\title{
SPAG6 regulates cell apoptosis through the TRAIL signal pathway in myelodysplastic syndromes
}

\author{
XINXIN LI, BIHUI YANG, LI WANG, LIPING CHEN, XIAOHUA LUO and LIN LIU \\ Department of Hematology, The First Affiliated Hospital of Chongqing Medical University, \\ Chongqing 400016, P.R. China
}

Received September 18, 2016; Accepted February 23, 2017

DOI: $10.3892 /$ or.2017.5540

\begin{abstract}
Myelodysplastic syndromes (MDSs) are a group of malignant clone hematopoietic stem-cell diseases, and the evolution and progression of MDS depend on the abnormal apoptosis of bone marrow cells. Our previous studies have indicated that sperm-associated antigen 6 (SPAG6), located in the uniparental disomy regions of myeloid cells, is overexpressed in patients with MDS as compared to controls, and SPAG6 can inhibit apoptosis of SKM-1. However, the concrete mechanism is still unclear. In the present study, it was found that the TNF-related apoptosis-inducing ligand (TRAIL) signal pathway was activated when the expression of SPAG6 was inhibited by SPAG6-shRNA lentivirus in SKM-1 cells. Additionally, the results of flow cytometry, Cell Counting Kit-8 assay and western blot analysis implied that the TRAIL signal pathway could be inhibited by a high expression of SPAG6. However, SPAG6 cannot influence the expression of TRAIL death receptors, except for FADD. Additionally the interaction between FADD and TRAIL death receptors also increased in SKM-1 cells infected with SPAG6-shRNA lentivirus. Thus, our study demonstrates that SPAG6 may regulate apoptosis in SKM-1 through the TRAIL signal pathway, indicating that SPAG6 could be a potential therapeutic target.
\end{abstract}

\section{Introduction}

Myelodysplastic syndromes (MDSs), one of the five major categories of myeloid neoplasms, are defined as clonal stem-cell disorders characterized by ineffective hematopoiesis in one or more of the lineages of the bone marrow, and the progression is from blood cytopaenias to acute myeloid leukaemia (AML) (1). AML, which is the most common type of acute leukemia in adults, is fatal in more than $50 \%$ of patients

Correspondence to: Dr Lin Liu, Department of Hematology, The First Affiliated Hospital of Chongqing Medical University, 1 Youyi Road, Yuzhong, Chongqing 400016, P.R. China

E-mail: liul7776@aliyun.com

Key words: myelodysplastic syndromes, SPAG6 gene, TRAIL, apoptosis, SKM-1 that do not receive current treatments (2), and the mortality of MDS is primarily caused by pancytopenia or transformation to AML. Therefore, the discovery of the molecular mechanisms would be significant for better prognoses. It has been discovered that MDS are one of the clonal haemopathies, in that an aberration within a haematopoietic progenitor cell gives rise to the entire disease (3). Additionally, some abnormal karyotypes have been found in the patients with MDS, including del (5q), monosomy 7 or del (7q), trisomy 8 and del (20q), whereas $\sim 50 \%$ patients have a normal karyotype (4-6).

The pathogenesis of MDS has not yet been completely elucidated. Recently, uniparental disomy (UPD) has garnered attention, and some studies have indicated that UPD is related to hematologic malignancies including both MDS and AML (7,8). Furthermore, sperm-associated antigen 6 (SPAG6) was shown to be overexpressed in some patients with MDS in a genome wide single nucleotide polymorphism analysis, which implies that SPAG6 may play a role in the pathogenesis of $\operatorname{MDS}(9)$.

SPAG6, which is the orthologue of Chlamydomonas PF16, is essential for sperm flagellar motility and maintenance of the structural integrity of mature sperm (10). In the last decade, several research groups have focused on the relationship between SPAG6 and malignancies. Seven genes, including WT1 and SPAG6, were found to be vastly overexpressed in patients with AML as compared to healthy bone marrow via genome-wide expression profiling (11). Furthermore, SPAG6 is highly expressed at the break point of $\mathrm{t}(10 ; 11)(\mathrm{p} 12 ; \mathrm{q} 14)$ in patients with CALM/AF10-positive leukemias (12). In our previous study, when the expression of SPAG6 was silenced by SPAG6-shRNA lentivirus, the growth of SKM-1 and K652 was markedly inhibited, and apoptosis was increased (13). The above evidence indicates that SPAG6 may promote cellular growth by preventing apoptosis. However, the specific mechanism of anti-apoptosis is still unknown.

Several studies imply that abnormal apoptosis is one of the underlying mechanisms in MDS, and tumor necrosis factor (TNF)- $\alpha$, Fas ligand, TNF-related apoptosis-inducing ligand (TRAIL) and other pro-apoptotic cytokines may be the major factors (14). In the last decades, the role of TRAIL in the pathogenesis of malignancies has been observed. TRAIL, also known as Apo2 ligand (Apo2L), is a member of the TNF ligand superfamily, and it is induced in tumor cells, but seldom 
in normal cells (15). Recently, two researchers explored the possible potential phenotype related to the TRAIL signal pathway using a genome-wide siRNA screen, and it was found that SPAG6 and this pathway may have a correlation, which was recorded in the GenomeRNAi-database (http://www. genomernai.org) (16).

The aim of this study is to clarify the regulatory mechanism of SPAG6 in cell apoptosis of MDS by TRAIL signal pathway, which may provide a new perspective in the development of MDS.

\section{Materials and methods}

Cell culture and infection. The human MDS cell line SKM-1, was kindly provided by Professor Zhou at the Tongji Medical College, Huazhong University of Science and Technology (Wuhan, China). The erythroleukemia K562 and the histiocytic lymphoma U937 cell lines were kept frozen in our laboratory. Cell line SKM-1, U937 and K562 were cultured in RPMI-1640 containing 10\% fetal bovine serum (FBS; Capricorn Scientific GmbH, Ebsdorfergrund, Germany) and no antibiotic in $5 \% \mathrm{CO}_{2}, 95 \%$ air incubator at $37^{\circ} \mathrm{C}$. For the infection protocol, SKM-1 cells at the exponential stage were plated in 6-well plates $\left(5 \times 10^{4}\right.$ cells/well), and were infected with the SPAG6-shRNA lentivirus or NC-shRNA lentivirus at a multiplicity of infection (MOI) of 20 in the concentration of $5 \mu \mathrm{g} / \mathrm{ml}$ polybrene, respectively. After $10 \mathrm{~h}$, the cells were washed and then resuspended in complete medium. After 5 days, the transfection efficiencies were evaluated by flow cytometry.

Cell treatment. SKM-1, K562 and U937 were seeded in 6 -well plates $\left(1 \times 10^{5}\right)$, and were treated with recombinant human sTRAIL (310-04; Peprotech, Rocky Hill, NJ, USA), respectively. The treated concentrations were as follows: 0 , 20, 40, 60, 80, 100 and $50 \mathrm{ng} / \mathrm{ml}$. After $24 \mathrm{~h}$, the cells were collected, and the apoptosis rates were measured by flow cytometry.

Flow cytometry. The apoptosis rates were detected using Annexin V and 7-ADD double-staining by flow cytometry. Following the cell treatments, the cells were collected and washed twice with phosphate-buffered saline (PBS). The early apoptotic cells contained Annexin $\mathrm{V}^{+} / \mathrm{PI}^{-}$, while the late apoptotic cells contained Annexin $\mathrm{V}^{+} / \mathrm{PI}^{+}$. Both of the two stages of apoptotic cells were counted, and the results were illustrated as a percentage of the total cell count. The techniques were supported by the Life Science Department of Chongqing Medical University (Chongqing, China).

RNA isolation and real-time PCR. The total cellular RNA from each group was extracted using the TRIzol reagent, and the reverse transcription reaction was performed using the Prime Script ${ }^{\mathrm{TM}}$ RT reagent kit (Takara Biotechnology Co., Ltd., Dalian, China). The cDNA was amplified in a $10-\mu 1$ PCR mix with $5 \mu 1$ of SYBR-Green super mixture. The PCR reactions were performed in a CFX-Connect Real-Time PCR system (Bio-Rad Laboratories Inc., Hercules, CA, USA). The cycling parameters were: $95^{\circ} \mathrm{C}$ for $30 \mathrm{sec}$, then 40 cycles at $95^{\circ} \mathrm{C}$ for $5 \mathrm{sec}$ and $60^{\circ} \mathrm{C}$ for $30 \mathrm{sec}$. PCR primers were as follows: SPAG6 forward, 5'-CCTTTCAGCTCTCAGTCAGG TTTC-3' and reverse, 5'-TCTTCACGTTTCATCCTTGTC CTT-3'; death receptor 4 (DR4) forward, 5'-TCGCTGTCCAC TTTCGTCT-3' and reverse, 5'-GGCGTTCCGTCCAGTT TTG-3'; DR5 forward, 5'-AAGACCCTTGTGCTCGTTGT-3' and reverse, 5'-GCTGCAACTGTGACTCCTAT-3'; and GAPDH forward, 5'-CTTTGGTATCGTGGAAGGACTC-3' and reverse, 5'-GTAGAGGCAGGGATGATGTTCT-3'. The PCR reactions were performed in a CFX-Connect Real-Time PCR system for 40 cycles (Bio-Rad Laboratories, Inc.). The relative gene expression levels were calculated by the $2^{-\Delta \Delta \mathrm{Ct}}$ method.

Protein isolation and western blot analysis. The cells were collected and lysed in RIPA lysis buffer (Beyotime, Beijing, China) containing $1 \mu \mathrm{M}$ PMSF, and the concentration was measured using the BCA Protein Assay kit (Beyotime). The total cell lysate was denatured via boiling and a total of $50 \mu \mathrm{g}$ of protein per lane was separated by sodium dodecyl sulfate-polyacrylamide gel electrophoresis (SDS-PAGE), and transferred to PVDF membranes. The membranes were blocked with $5 \%$ bovine serum albumin for $90 \mathrm{~min}$ at room temperature and then incubated overnight at $4{ }^{\circ} \mathrm{C}$ with specific antibodies. The primary antibodies were as follows: GAPDH (AG019, 1:1,000; Beyotime) cleaved caspase-8 (9496, 1:500; Cell Signaling Technology, Inc., Beverly, MA, USA); SPAG6, PARP, cleaved PARP, caspase-8, DR4, DR5 and FADD (ab155653, ab32138, ab32561, ab32397, ab8414, ab181846, ab108601, 1:1,000; all from Abcam, Cambridge, UK); caspase-3 and BID (YT0656, YT0488, 1:500; both from Immunoway, Newark, DE, USA); and BAX and BAK (RLT0456, RLT0449, 1:500; both from Ruiying Biological, Suzhou, China). After five washes with TBS-Tween-20, the membranes were incubated with a goat anti-rabbit of goat anti-mouse peroxidase-conjugated second antibody (A0216, A0208, 1:1,000; Beyotime) for $1 \mathrm{~h}$ at $37^{\circ} \mathrm{C}$. Then, the excess antibody was removed from the blots with TBS-Tween-20 three times before incubation in ECL. The protein expression levels were analysed using Vilber Fusion software (Fusion FX5 Spectra; Vilber Lourmat, Marne-La-Vallée, France).

The Cell Counting Kit-8 (CCK-8) assay. Apoptosis was measured by a CCK-8 assay. Cells in exponential growth were seeded in 96 -well plates at a density of $8 \times 10^{3}$ cells/well, and then, they were treated with recombinant human sTRAIL (rTRAIL) containing different concentrations for $24 \mathrm{~h}$, respectively. Finally, $10 \mu 1$ of CCK-8 (Dojindo Laboratories, Kumamoto, Japan) solution was added to each well, and the plates were incubated at $37^{\circ} \mathrm{C}$ for $100 \mathrm{~min}$. Cell apoptosis was estimated by measuring the absorbance at $450 \mathrm{~nm}$ using the plate reader. All conditions were tested in five replicates.

Immunoprecipitation. The cells infected with the lentivirus were collected after infection and lysed in RIPA lysis buffer. The solution was centrifuged at $12,000 \mathrm{rpm}$ for $10 \mathrm{~min}$ at $4^{\circ} \mathrm{C}$ and the supernatants were kept. Protein A agarose and antibody (ab108601, 1:100, ab14738, ab181846, 1:2,000; Abcam) diluted with RIPA buffer were mixed and incubated overnight at $4^{\circ} \mathrm{C}$. Then the total protein was mixed and incubated overnight at $4^{\circ} \mathrm{C}$. After incubation, the mixture was washed six 
A
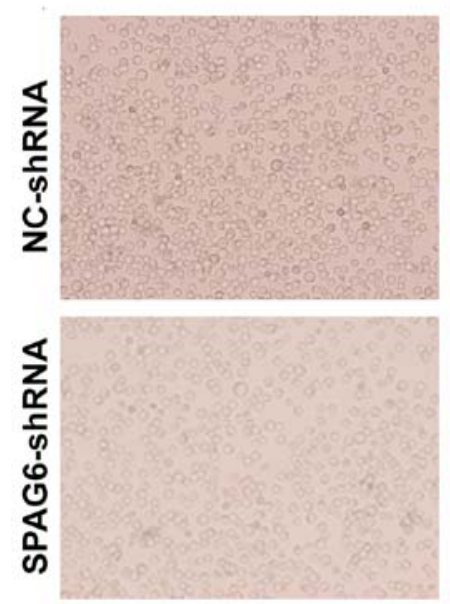

C

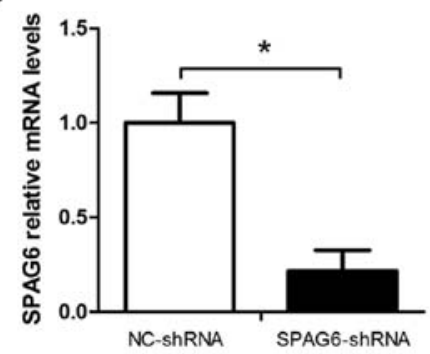

B
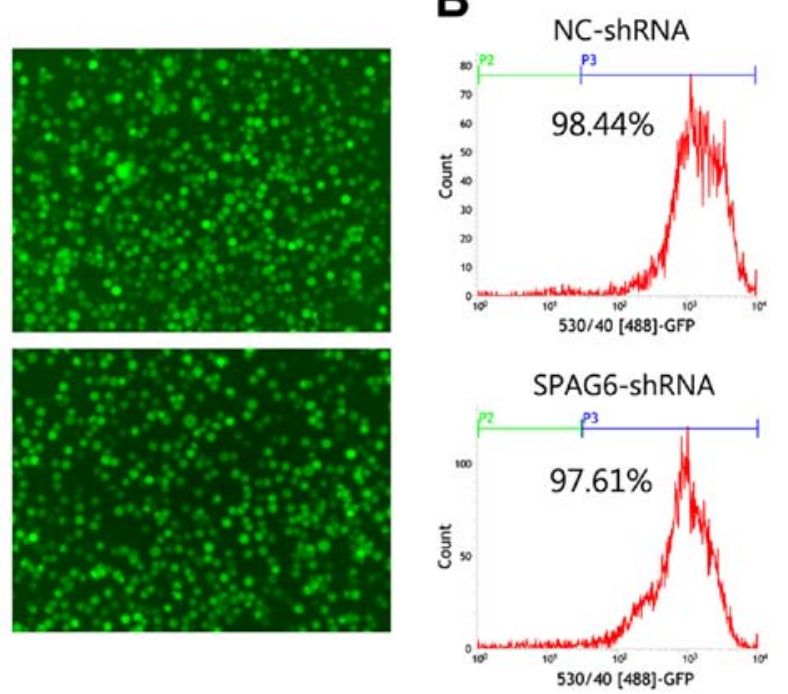

D

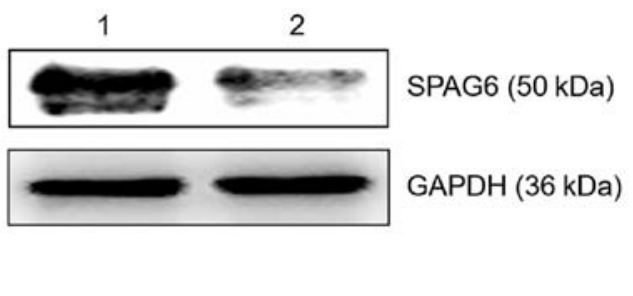

Figure 1. Knockdown of SPAG6 expression by lentivirus. (A) SKM-1 cells infected with lentivirus were observed under a fluorescence microscope (x40 magnification). (B) Transfection efficiencies were detected by flow cytometry. (C) SPAG6 expression was inhibited at the mRNA level. (D) Protein expression of SPAG6 was measured by western blot analysis, and GAPDH was used as the internal reference. ${ }^{*}$ P $<0.05$. Lane 1, NC-shRNA group; lane 2, SPAG6-shRNA group. SPAG6, sperm-associated antigen 6.

times with different types of washing buffer. Finally, a western blot analysis was performed for immunoblot analysis.

Statistical analysis. All the results were technically repeated three times, and were expressed as the mean \pm standard deviation (SD). The significant differences between groups were assessed by one-way analysis of variance (ANOVA) and Student's t-test (SPSS version 20.0; SPSS, Inc., Chicago, IL, USA) and a value of $\mathrm{P}<0.05$ was considered statistically significant.

\section{Results}

Lentivirus-mediated SPAG6 silence in SKM-1 cells. The lentivirus expressing shRNA was used against SPAG6 for gene silencing in SKM-1 cells, and the SKM-1 cells infected with NC-shRNA lentivirus were used as a control group. Six days after infection, abundance of cells with green fluorescence could be observed under a fluorescence microscope (Fig. 1A). The transfection efficiencies were detected by FACS, and the results were $97.19 \pm 3.21 \%$ and $88.31 \pm 12.81 \%$ (Fig. 1B). This demonstrated that the SKM-1 cells were successfully infected by the lentivirus. The inhibitory degree of SPAG6 was measured by qRT-PCR and western blot analysis. The results showed that both the mRNA and protein levels were reduced in cells infected with SPAG6 shRNA-lentivirus as compared to the control group (Fig. 1C and D).
Reducing the expression of SPAG6 activates the TRAIL signal pathway. Apoptosis was analysed by Annexin V and 7-AAD assays, as illustrated in Fig. 2A and B, and the apoptosis rates in SKM-1 infected with SPAG6-shRNA were significantly higher than those in the control group (NC-shRNA, 8.65 $\pm 2.07 \%$ vs. SPAG6-shRNA, 20.20 $\pm 2.11 \%$; $\mathrm{P}<0.05)$. To explore the activation of the TRAIL signal pathway, the expression of related proteins was examined. Western blot analysis revealed that the expression levels of cleaved PARP, caspase- 8 and cleaved caspase- 8 were obviously higher in SKM-1 infected with SPAG6-shRNA lentivirus than in the control group, whereas the expression of PARP showed no significant difference between the two groups (Fig. 2C and D). Furthermore, the expression of apoptotic factors, including BAK, BAX, BID and caspase-3, also showed statistical differences in the two groups (Fig. 3E and F). These data indicate that the TRAIL signal pathway was activated when the expression of SPAG6 was reduced.

SPAG6 resists apoptosis induced by TRAIL. Following rTRAIL treatment, the percentage of apoptosis cells increased, and the flow cytometry data indicated that the higher concentration of TRAIL led to higher apoptosis rates (Fig. 3A and B). To investigate the growth of SKM-1 cells treated with different concentrations of TRAIL, CCK-8 assays were performed to analyse cell proliferation. Cell proliferation was suppressed in the cells treated with TRAIL as compared to the normal 
A
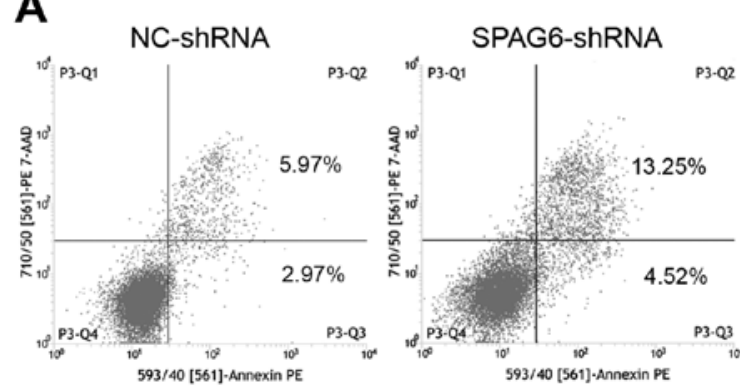

C

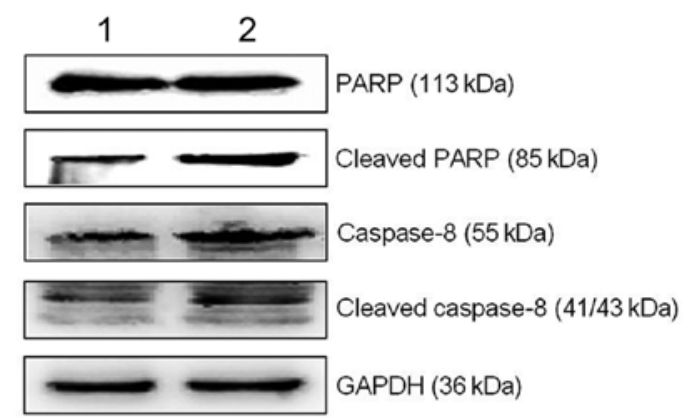

E

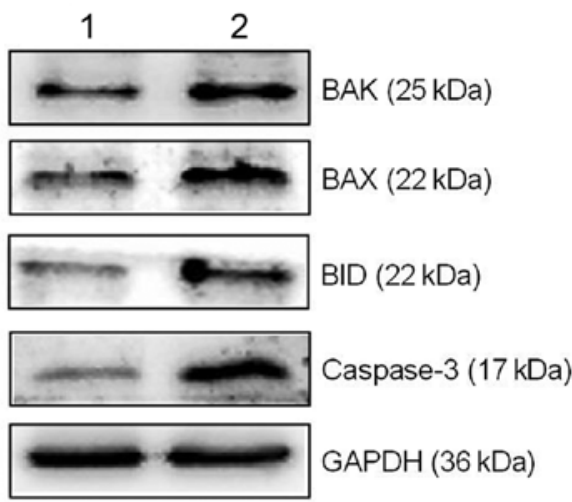

B

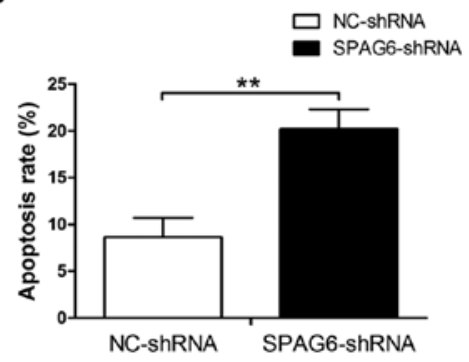

D

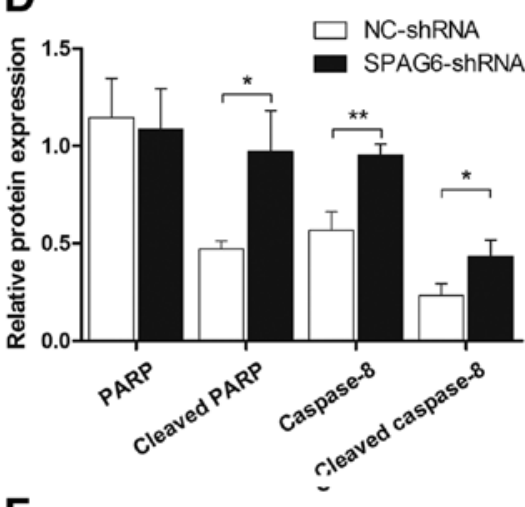

$\mathbf{F}$

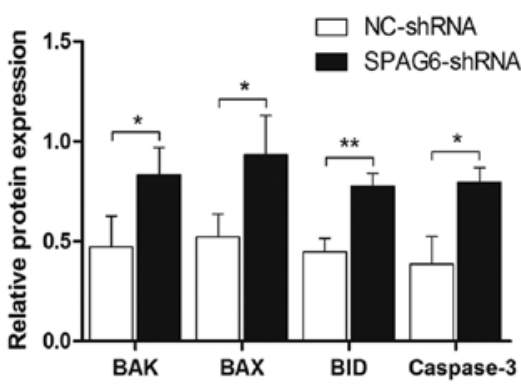

Figure 2. TRAIL signal pathway is activated when the expression of SPAG6 is inhibited. (A) After transfection with lentivirus, the apoptosis rate was evaluated by flow cytometry. (B) The analysis of the apoptosis rates is shown in (A). The apoptosis rate of SKM-1 cells infected with SPAG6-shRNA significantly increased as compared to cells infected with NC-shRNA. (C) Expression of PARP, cleaved PARP, caspase-8 and cleaved caspase-8 protein was detected by western blot analysis. (D) TRAIL signal pathway was activated. (E) Expression of BAK, BAX, BID and caspase-3 protein was detected by western blot analysis. (F) These proteins showed statistical differences between the groups. The data are shown as mean \pm SD. ${ }^{*} \mathrm{P}<0.05,{ }^{* *} \mathrm{P}<0.01$. Lane $1, \mathrm{NC}$-shRNA group; lane 2, SPAG6-shRNA group. TRAIL, TNF-related apoptosis-inducing ligand; SPAG6, sperm-associated antigen 6.

control group (Fig. 3C). In addition, western blot analysis showed that the expression of related proteins was higher after treatment (Fig. 3D and E). However, as shown in Fig. 3F and G, in the SKM-1, U937 and K562 cells after treatment with the same concentration of TRAIL, the apoptosis rates were not significantly different except for that of SKM-1 (the expression of SPAG6, SKM-1<U937<K562), and similar results were also observed in the CCK-8 assays (Fig. $3 \mathrm{H}$ ). These data illustrate that a high expression of SPAG6 may resist apoptosis induced by TRAIL.

SPAG6 has no effect on the expression of TRAIL death receptors, except for FADD. TRAIL induces apoptosis through DR4 (TNFRSF10A) and DR5 (TNFRSF10B). Therefore, the degree of apoptosis induced by TRAIL is closely related to the levels of DR4 and DR5. As illustrated in the figure, both the mRNA and protein levels of the two receptors were not changed (Fig. 4A-C). However, the expression of FADD was statistically different (Fig. 4B and C), indicating that the regulation of apoptosis may not be through DR4 and DR5 expression.

SPAG6 influences the interaction between the FADD and TRAIL death receptors. As shown in the Fig. 4, the expressions of DR4 and DR5 showed no difference. Therefore, immunoprecipitation was used to explore the relationship between the FADD and TRAIL death receptors. FADD was used as the bait protein to immunoprecipitate DR4 and DR5. Then, DR4 and DR5 were blotted. As shown in the figure, the interaction between FADD and both death receptors increased in SKM-1 infected with SPAG6 shRNA-lentivirus as compared to the control group (Fig. 4D). 
A
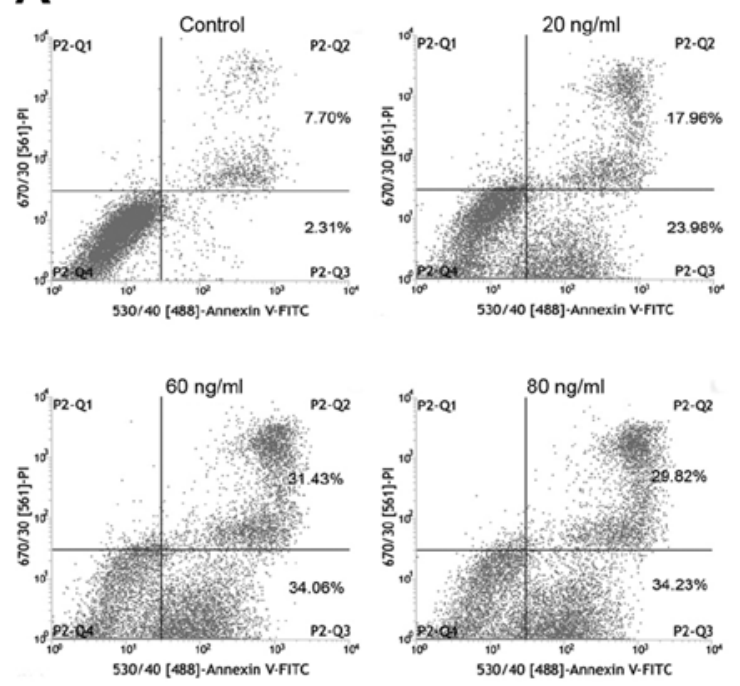

D

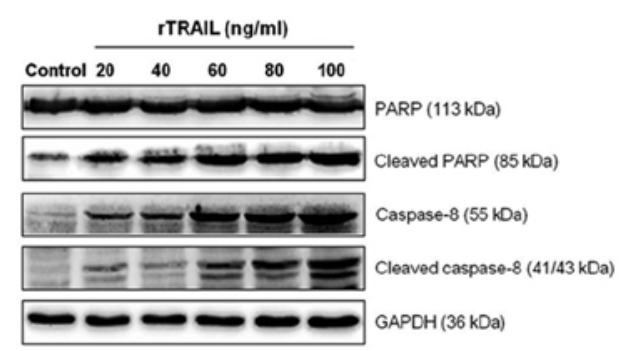

$\mathbf{F}$
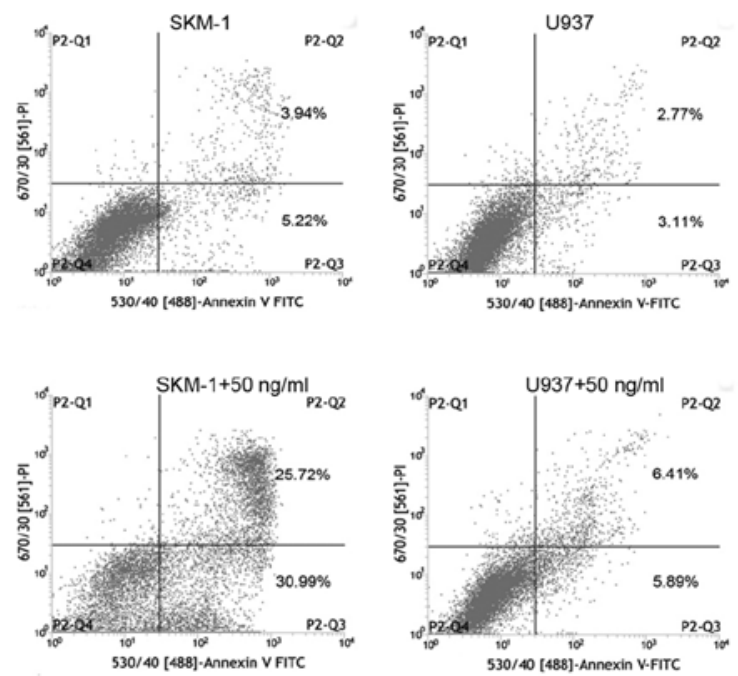

E

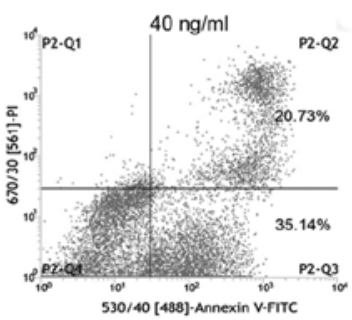

B


C
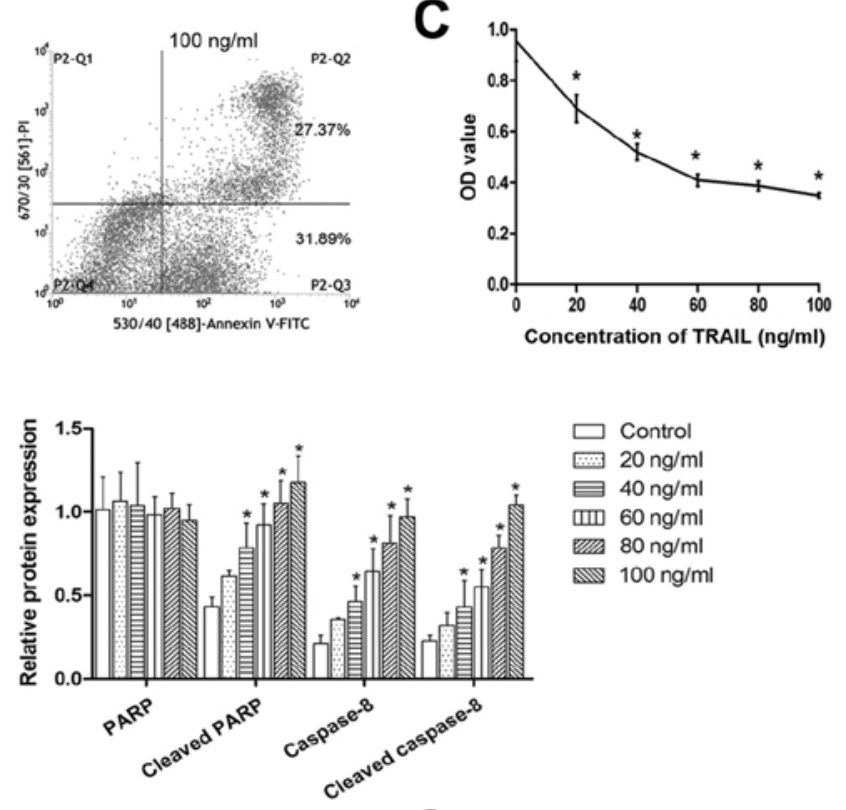

G
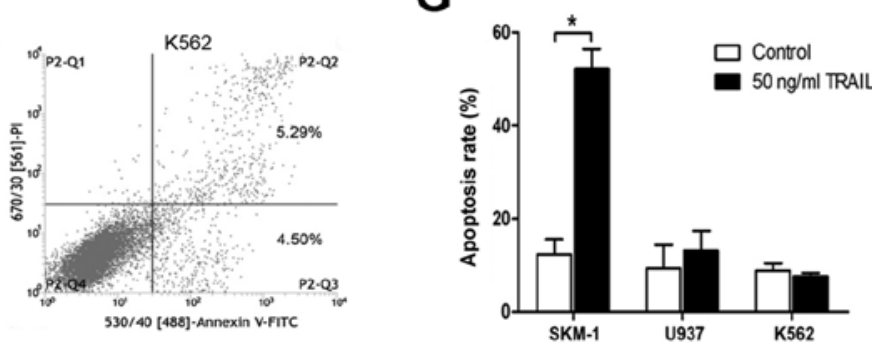

H
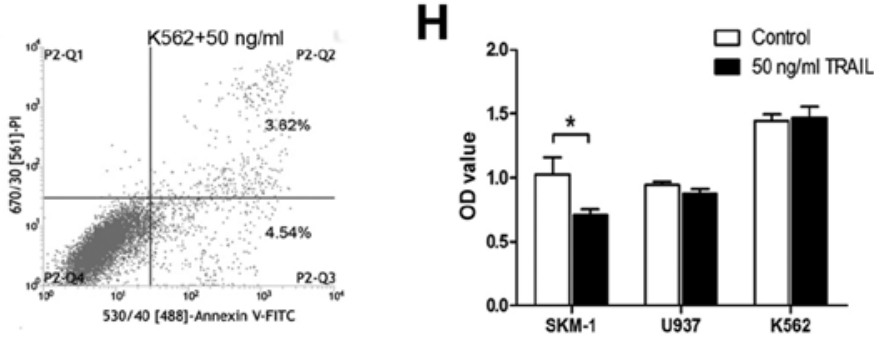

Figure 3. SPAG6 may resist apoptosis induced by TRAIL. (A) Following rTRAIL treatment, the apoptosis rates of SKM-1 cells were detected by flow cytometry. (B) A higher concentration of rTRAIL led to higher apoptosis rates in SKM-1 cells. (C) The proliferation was assessed by a CCK-8 assay. (D) PARP, cleaved PARP, caspase- 8 and cleaved caspase- 8 protein expression were detected by western blot analysis. (E) The analysis of the relative protein expression. (F) After SKM-1, U937 and K562 cells were treated with the same concentration of TRAIL, the apoptosis rates were measured by flow cytometry. (G) The apoptosis rates were not significantly different in the U937 and K562 cells (the expression of SPAG6, SKM-1<U937<K562; https://portals.broadinstitute. org/ccle/home). (H) The proliferation showed the same results as the apoptosis rates. The data are shown as mean \pm SD. *P<0.05. SPAG6, sperm-associated antigen 6; TRAIL, TNF-related apoptosis-inducing ligand.

\section{Discussion}

Apoptosis is a process of programmed cell death, which can occur in multicellular organisms in order to maintain homeostasis. In brief, apoptosis is initiated through two pathways, the extrinsic pathway and the intrinsic pathway mediated by mitochondrion (17). Abnormal apoptosis, including resistance to apoptosis, is regarded as an important mechanism of tumorigenesis (18). MDS is characterized by ineffective hematopoiesis leading to peripheral blood 
A

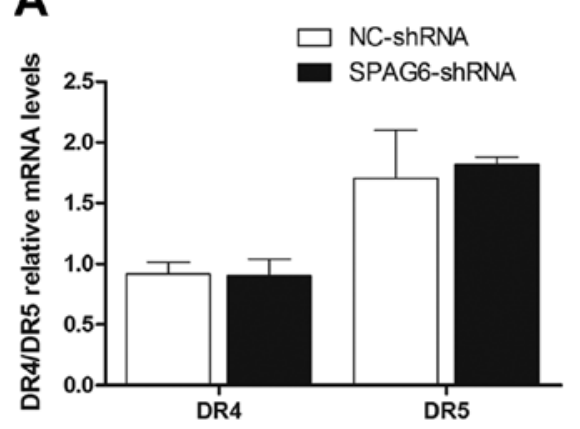

C

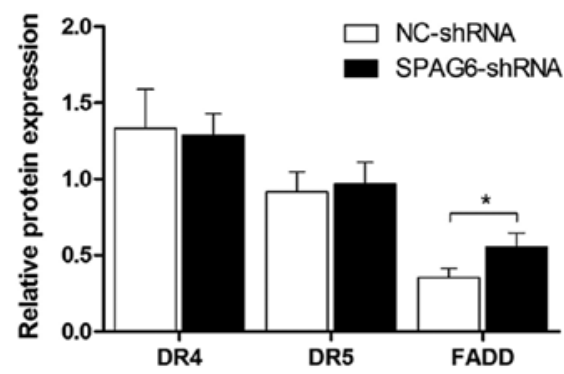

B

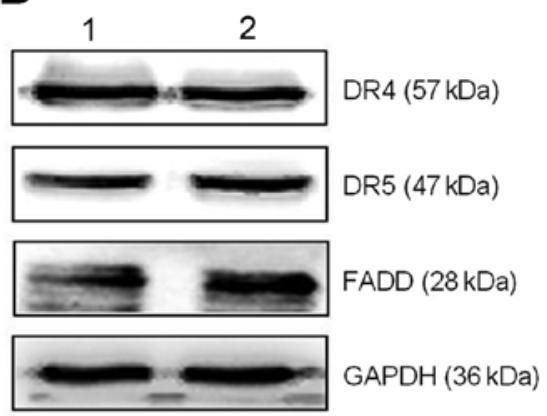

D

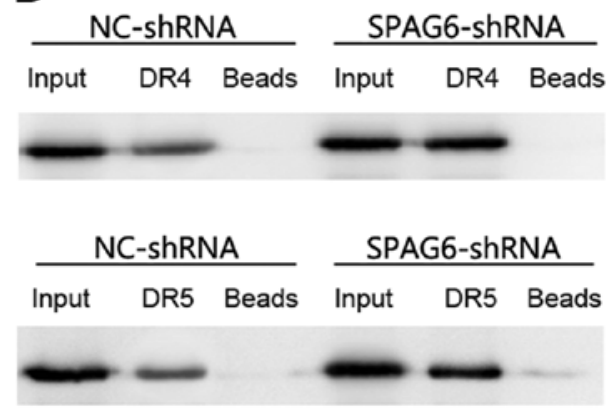

Figure 4. SPAG6 can influence the interaction between FADD and TRAIL death receptors. (A) The mRNA expression of DR4 and DR5 showed no statistical difference. (B) The DR4, DR5 and FADD protein expression were determined by western blot analysis. (C) SPAG6 showed no effect on the expression of TRAIL death receptors, except for FADD. (D) Immunoprecipitation was used to explore the interaction between FADD and TRAIL death receptors. The interaction between FADD and death receptors increased in SKM-1 cells infected with SPAG6-shRNA lentivirus. The data are shown as mean \pm SD. ${ }^{*}$ P $<0.05$. Lane 1, NC-shRNA group; lane 2, SPAG6-shRNA group. SPAG6, sperm-associated antigen 6; TRAIL, TNF-related apoptosis-inducing ligand.

cytopenia, and one of the possible mechanisms is excessive apoptosis in hematopoietic precursors. It has been found that TRAIL and its receptors are expressed at a low level in normal marrow, and the opposite occurs in MDS marrow cells (19). Furthermore, the expression of TRAIL signal inhibitor including cFLIP, XIAP and the Bcl-2 family, is higher in patients with MDS in advanced stages than those with MDS in the early stages (20-22). The evidence indicates that the TRAIL signal pathway may play a role in the development of MDS.

SPAG6 was first detected in human testis tissue. Its major functions are participation in the maturation of reproductive cells and maintaining sperm motility and fertility $(10,23,24)$. Recent studies have found that SPAG6 is upregulated in CALM/AF10-positive leukemia and pediatric AML $(11,12)$. Additionally, a prospective multicenter study indicated that patients with AML may obtain a better prognosis and relapse-free survival (RFS) when SPAG6 is expressed at a low level (25). Furthermore, in lung and breast cancer, SPAG6 is defined as a novel cancer-testis (CT) antigen, which can be considered as a tumor marker and potential candidate for use in cancer therapy (26), implying that SPAG6 may lead to carcinogenesis and can be a parameter for assessing their efficacy or prognosis. However, the molecular mechanism of SPAG6 in hematologic malignancy has seldom been studied.

Therefore, in the present study, we explored the relationship between SPAG6 and the TRAIL signal pathway to illustrate the possible mechanism of the regulation of apoptosis in SKM-1. TRAIL can induce apoptosis in cells through its receptors at the cell surface, DR4 and DR5 (27). After TRAIL binds to receptors, the adapter protein FADD is recruited through cytoplasmic death domains, and then, FADD interacts with pro-caspase- 8 recruited to form the death-inducing signaling complex (DISC). DISC then mediates the activation of pro-caspase- 8 (28). Caspase- 8 directly activates caspase- 3 to cleave the substrates, such as PARP (29). Therefore, the activation of caspase- 8 and the cleavage of PARP are chosen as parameters to measure apoptosis. Moreover, BID, a target of active caspase- 8 , is cleaved to form truncated BID (tBID) and tBID then leads the participation of mitochondrial apoptotic factors (30). As is shown in the results, after the expression of SPAG6 was reduced by the lentivirus, the apoptosis rates increased, and the TRAIL signal pathway was activated, and both the intrinsic and extrinsic pathways were triggered. This results suggests that SPAG6 may affect apoptosis through the TRAIL signaling pathway.

A preliminary study indicated that a high expression of SPAG6 is connected with apoptosis resistance in SKM-1 and K562 (13). In this study, when SKM-1 cells were treated with different concentrations of rTRAIL, the apoptosis rates showed an increased tendency. However, given the same concentration of rTRAIL, cells with high expression levels of SPAG6 exhibited insensitivity to apoptosis induced by rTRAIL. In addition, the expression of DR4 and DR5 showed no differences at the mRNA and protein levels, but the expression of FADD increased. These data imply that the regulation may not occur through the receptors. In order to explore the possible mechanism, immunoprecipitation was used to measure the interaction between FADD and both DR4 and DR5. It was found that the interaction between FADD and DR4 and that 
between FADD and DR5 both increased. Moreover, p53 is the most commonly mutated gene in human cancer (31), and large quantities of studies have demonstrated its anticancer functions. Additionally, some studies have shown that p53 may also participate in the regulation of apoptosis induced by TRAIL, and the possible approaches are to influence the expression of the TRAIL receptors $(32,33)$ or the BCL-2 family (34). In addition, TRAIL can induce not only the initiation of apoptosis but also the non-apoptotic pathways, such as nuclear factor- $\kappa \mathrm{B}(\mathrm{NF}-\kappa \mathrm{B})$, phosphatidylinositol 3-kinase (PI3K) and Akt, and mitogen activated protein kinases (MAPKs) (35). It is important to note that these studies are performed on the cellular level, so an in vivo study is needed to confirm the results. Furthermore, there are limitations inherent in using a cell line in culture versus using primary bone marrow cells from patients. Therefore, further studies are needed to explore the possible mechanisms.

In conclusion, this study demonstrates that SPAG6 may regulate apoptosis through the TRAIL signal pathway by inhibiting the expression of FADD and the interactions between FADD and the TRAIL death receptors. TRAIL has been proven to possess anticancer functions. In animal models, it has shown that the growth of a TRAIL-sensitive tumor is suppressed by rTRAIL without significant system toxicity $(36,37)$. MDS treatments have rapidly improved; however, there is still lack of effective methods, and antileukemic drugs can enhance apoptosis mediated by TRAIL (38). The above may indicate a new therapy for MDS. Therefore, SPAG6 may be a potential target in therapy for MDS, and it may also be worthwhile to explore drugs against SPAG6 function.

\section{Acknowledgements}

We thank Mr. Nian Zhou, Ms. Xingyi Kuang and Mr. Hongrong Zhang for their valuable assistance. We would like to acknowledge the service provided by Laboratory of Lipid and Glucose Metabolism, the First Affiliated Hospital of Chongqing Medical University, Chongqing, China. This study is supported by the Natural Science Foundation of Chongqing (no. CSTC2013jjB10020) and the National Natural Sciences Foundation of China (no. 81570109).

\section{References}

1. Corey SJ, Minden MD, Barber DL, Kantarjian H, Wang JC and Schimmer AD: Myelodysplastic syndromes: the complexity of stem-cell diseases. Nat Rev Cancer 7: 118-129, 2007.

2. Wouters BJ and Delwel R: Epigenetics and approaches to targeted epigenetic therapy in acute myeloid leukemia. Blood 127 42-52, 2016.

3. Shih AH, Abdel-Wahab O, Patel JP and Levine RL: The role of mutations in epigenetic regulators in myeloid malignancies. Nat Rev Cancer 12: 599-612, 2012.

4. Haase D, Germing U, Schanz J, Pfeilstöcker M, Nösslinger T, Hildebrandt B, Kundgen A, Lübbert M, Kunzmann R, Giagounidis AA, et al: New insights into the prognostic impact of the karyotype in MDS and correlation with subtypes: evidence from a core dataset of 2124 patients. Blood 110: 4385-4395, 2007.

5. Bernasconi P, Klersy C, Boni M, Cavigliano PM, Calatroni S, Giardini I, Rocca B,Zappatore R, Caresana M, Dambruoso I, et al: World Health Organization classification in combination with cytogenetic markers improves the prognostic stratification of patients with de novo primary myelodysplastic syndromes. Br J Haematol 137: 193-205, 2007.
6. Schanz J, Steidl C, Fonatsch C, Pfeilstöcker M, Nösslinger T, Tuechler H, Valent P, Hildebrandt B, Giagounidis A, Aul C, et al: Coalesced multicentric analysis of 2,351 patients with myelodysplastic syndromes indicates an underestimation of poor-risk cytogenetics of myelodysplastic syndromes in the international prognostic scoring system. J Clin Oncol 29: 1963-1970, 2011.

7. Maciejewski JP and Mufti GJ: Whole genome scanning as a cytogenetic tool in hematologic malignancies. Blood 112: 965-974, 2008

8. Kralovics R: Genetic complexity of myeloproliferative neoplasms. Leukemia 22: 1841-1848, 2008.

9. Wang L, Fidler C, Nadig N, Giagounidis A, Della Porta MG, Malcovati L, Killick S, Gattermann N, Aul C, Boultwood J, et al: Genome-wide analysis of copy number changes and loss of heterozygosity in myelodysplastic syndrome with del(5q) using high-density single nucleotide polymorphism arrays. Haematologica 93: 994-1000, 2008.

10. Sapiro R, Kostetskii I, Olds-Clarke P, Gerton GL, Radice GL and Strauss JF III: Male infertility, impaired sperm motility, and hydrocephalus in mice deficient in sperm-associated antigen 6. Mol Cell Biol 22: 6298-6305, 2002.

11. Steinbach D, Schramm A, Eggert A, Onda M, Dawczynski K, Rump A, Pastan I, Wittig S, Pfaffendorf N, Voigt A, et al: Identification of a set of seven genes for the monitoring of minimal residual disease in pediatric acute myeloid leukemia. Clin Cancer Res 12: 2434-2441, 2006.

12. Mulaw MA, Krause A, Deshpande AJ, Krause LF, Rouhi A, La Starza R, Borkhardt A, Buske C, Mecucci C, Ludwig WD, et al: $C A L M / A F 10$-positive leukemias show upregulation of genes involved in chromatin assembly and DNA repair processes and of genes adjacent to the breakpoint at 10p12. Leukemia 26: 1012-1019, 2012.

13. Yang B, Wang L, Luo X, Chen L, Yang Z and Liu L: SPAG6 silencing inhibits the growth of the malignant myeloid cell lines SKM-1 and K562 via activating p53 and caspase activation-dependent apoptosis. Int J Oncol 46: 649-656, 2015.

14. Kerbauy DB and Deeg HJ: Apoptosis and antiapoptotic mechanisms in the progression of myelodysplastic syndrome. Exp Hematol 35: 1739-1746, 2007.

15. Griffith TS and Lynch DH: TRAIL: a molecule with multiple receptors and control mechanisms. Curr Opin Immunol 10: $559-563,1998$

16. Kranz D and Boutros M: A synthetic lethal screen identifies FAT1 as an antagonist of caspase- 8 in extrinsic apoptosis. EMBO J 33: 181-197, 2014.

17. Igney FH and Krammer PH: Death and anti-death: tumour resistance to apoptosis. Nat Rev Cancer 2: 277-288, 2002.

18. Martin GS: Cell signaling and cancer. Cancer Cell 4: 167-174, 2003.

19. Zang DY, Goodwin RG, Loken MR, Bryant E and Deeg HJ: Expression of tumor necrosis factor-related apoptosis-inducing ligand, Apo2L, and its receptors in myelodysplastic syndrome: effects on in vitro hemopoiesis. Blood 98: 3058-3065, 2001.

20. Benesch M, Platzbecker U, Ward J, Deeg HJ and Leisenring W: Expression of FLIP(Long) and FLIP(Short) in bone marrow mononuclear and $\mathrm{CD}_{3} 4^{+}$cells in patients with myelodysplastic syndrome: correlation with apoptosis. Leukemia 17: 2460-2466, 2003.

21. Yamamoto K, Abe S, Nakagawa Y, Suzuki K, Hasegawa M, Inoue M, Kurata M, Hirokawa $\mathrm{K}$ and Kitagawa M: Expression of IAP family proteins in myelodysplastic syndromes transforming to overt leukemia. Leuk Res 28: 1203-1211, 2004.

22. Parker JE, Mufti GJ, Rasool F, Mijovic A, Devereux S and Pagliuca A: The role of apoptosis, proliferation, and the Bcl-2-related proteins in the myelodysplastic syndromes and acute myeloid leukemia secondary to MDS. Blood 96: 3932-3938, 2000.

23. Zhang Z, Jones BH, Tang W, Moss SB, Wei Z, Ho C, Pollack M, Horowitz E, Bennett J, Baker ME, et al: Dissecting the axoneme interactome: the mammalian orthologue of Chlamydomonas PF6 interacts with sperm-associated antigen 6, the mammalian orthologue of Chlamydomonas PF16. Mol Cell Proteomics 4: 914-923, 2005.

24. Pearson CG, Giddings TH Jr and Winey M: Basal body components exhibit differential protein dynamics during nascent basal body assembly. Mol Biol Cell 20: 904-914, 2009.

25. Steinbach D, Bader P, Willasch A, Bartholomae S, Debatin KM, Zimmermann M, Creutzig U, Reinhardt D and Gruhn B: Prospective validation of a new method of monitoring minimal residual disease in childhood acute myelogenous leukemia. Clin Cancer Res 21: 1353-1359, 2015. 
26. Silina K, Zayakin P, Kalnina Z, Ivanova L, Meistere I, Endzelins E, Abols A, Stengrēvics A, Leja M, Ducena K, et al: Sperm-associated antigens as targets for cancer immunotherapy: expression pattern and humoral immune response in cancer patients. J Immunother 34: 28-44, 2011.

27. Ashkenazi A and Dixit VM: Death receptors: signaling and modulation. Science 281: 1305-1308, 1998.

28. Scaffidi C, Kirchhoff S, Krammer PH and Peter ME: Apoptosis signaling in lymphocytes. Curr Opin Immunol 11: 277-285, 1999.

29. Gonzalvez F and Ashkenazi A: New insights into apoptosis signaling by Apo2L/TRAIL. Oncogene 29: 4752-4765, 2010

30. Li H, Zhu H, Xu CJ and Yuan J: Cleavage of BID by caspase 8 mediates the mitochondrial damage in the Fas pathway of apoptosis. Cell 94: 491-501, 1998.

31. Kandoth C, McLellan MD, Vandin F, Ye K, Niu B, Lu C, Xie M, Zhang Q, McMichael JF, Wyczalkowski MA, et al: Mutational landscape and significance across 12 major cancer types. Nature 502: 333-339, 2013.

32. Liu X, Yue P, Khuri FR and Sun SY: p53 upregulates death receptor 4 expression through an intronic p53 binding site Cancer Res 64: 5078-5083, 2004.
33. Takimoto R and El-Deiry WS: Wild-type p53 transactivates the KILLER/DR5 gene through an intronic sequence-specific DNA-binding site. Oncogene 19: 1735-1743, 2000.

34. Henry H, Thomas A, Shen Y and White E: Regulation of the mitochondrial checkpoint in p53-mediated apoptosis confers resistance to cell death. Oncogene 21: 748-760, 2002.

35. Falschlehner $\mathrm{C}$, Emmerich $\mathrm{CH}$, Gerlach B and Walczak $\mathrm{H}$ : TRAIL signalling: decisions between life and death. Int $\mathrm{J}$ Biochem Cell Biol 39: 1462-1475, 2007.

36. Ashkenazi A, Pai RC, Fong S, Leung S, Lawrence DA, Marsters SA, Blackie C, Chang L, McMurtrey AE, Hebert A, et al: Safety and antitumor activity of recombinant soluble Apo2 ligand. J Clin Invest 104: 155-162, 1999.

37. Walczak H, Miller RE, Ariail K, Gliniak B, Griffith TS, Kubin M, Chin W, Jones J, Woodward A, Le T, et al: Tumoricidal activity of tumor necrosis factor-related apoptosis-inducing ligand in vivo. Nat Med 5: 157-163, 1999.

38. Wen J, Ramadevi N, Nguyen D, Perkins C, Worthington E and Bhalla K: Antileukemic drugs increase death receptor 5 levels and enhance Apo-2L-induced apoptosis of human acute leukemia cells. Blood 96: 3900-3906, 2000. 